\title{
CLINIQUIZ
}

CONCURRENT DISEASES

\section{Ischemic heart disease}

Priyanka Sehrawat ${ }^{1}$, Pranav Bansal ${ }^{2}$

1- Resident; 2- Professor \& Head, Department of Anesthesiology \& Critical Care, BPS Govt. Medical College, Khanpur Kalan, Sonepat, Haryana, India; E-mail: pranavbansal1@gmail.com

With a growing population of old people and the changes in the life style have increased the frequency of the anesthesiologist's encounters with surgical patients who have ischemic heart disease as a comorbid condition. Here are some questions related to ischemic heart disease. (Please tick the most appropriate option).

Q 1. Which of the following statement regarding right ventricular infarction is false:-
A. It usually signifies occlusion in right coronary artery
B. Right atrial pressure is $<10 \mathrm{mmHg}$
C. Inferior wall myocardial infarction is usually present
D. Right to left shunting can be seen

$Q$ 2.A patient in ICU is on low dose aspirin following myocardial infarction 2 years back. Which of the following statement is false:-
A. Aspirin irreversibly inhibits platelet cyclo- oxygenase
B. Aspirin may potentiate the effect of warfarin
C. In high doses, aspirin is more effective analgesic than most NSAIDs
D. Overdose can be treated with hemodialysis

Q 3. According to the Lee Revised Cardiac Risk Index (LCRI), which of the following is not a predictor of cardiac complications for patients undergoing major non cardiac surgery:-
A. History of ischemic heart disease
B. History of congestive cardiac failure
C. History of cerebrovascular disease
D. History of non-insulin dependent diabetes mellitus

Q 4. Recommended PCI-to- any elective surgery interval should be preferably:
A. Bare-metal stent $>30$ days, drug eluting stent $>2$ years
B. Bare metal stent $>45$ days, drug eluting stent $>1$ year
C. Bare metal stent $>90$ days, drug eluting stent $>1$ year
D. Bare metal stent $>90$ days, drug eluting stent $>$ 2 yrs

Q 5. Which of the following best distinguishes nonST-segment elevation myocardial infarction (NSTEMI) from unstable angina:-
A. Severe chest pain
B. ST depression in two or more contagious leads in ECG
C. Chest pain lasting more than $15 \mathrm{~min}$
D. Raised troponin level

Q 6. Which of the following statement is false with respect to reperfusion therapy:-
A. Should be initiated within 2-3 hrs of hospital arrival
B. Should be initiated within $12 \mathrm{hrs}$ of symptom onset
C. Intracranial hemorrhage is the most feared complication
D. Contraindicated in case of internal bleeding within 3 weeks

Q 7. Which of the following is not a prognostic determinant in patients with coronary artery disease:-
A. Resting heart rate
B. The state of left ventricular function 
C. Left main coronary artery stenosis greater than $50 \%$

D. Plague with thin fibrous cap and large lipid core

Q 8. A 70-yr old lady presents after few days following an anterior myocardial infarction with shortness of breath. Chest x-ray shows pulmonary venous congestion. Neck veins distended, systolic murmur is audible, hemodynamically hypotension. A pulmonary artery catheter is inserted, pulmonary artery pressure $40 / 21 \mathrm{mmHg}$ with pulmonary artery oxygen saturation is $85 \%$ are noted. Which of the following statement is incorrect:-

A. Doppler echocardiography is diagnostic

B. Medical management includes afterload reduction

C. Finding suggests left to right shunt

D. Papillary muscle rupture is the probable diagnosis

Q 9. Which of the following statement is false regarding intraoperative monitoring for

\section{myocardial ischemia:-}

A. Monitoring of lead V5 helps in identifying perioperative ischemia

B. Monitoring of Lead II helps in identifying perioperative arrhythmias

C. Pulmonary artery catheter monitoring is a sensitive method for intraoperative myocardial ischemia and manifest as acute increase in pulmonary artery occlusion pressure

D. New regional ventricular motion abnormalities on TEE is standard for diagnosing intraoperative MI

\section{Q 10.Which of the following statement is false} regarding transplanted heart:
A. It has no sympathetic or parasympathetic innervations
B. Resting heart rate is higher than normal
C. Responds to circulating catecholamines
D. Responds to atropine and ephedrine

\section{EXPLANATIONS:}

A 1 (B): Right ventricular infarction is usually accompanied with inferior infarction. The posterior descending branch of the right coronary artery supplies the inferior and posterior walls of the right ventricle. Right to left shunting can occur at the atrial level through a patent foramen ovale in the presence of elevated right atrial pressure (greater than 10 $\mathrm{mmHg}$ ).

A 2 (C): Aspirin in low dose selectively inhibits platelet $\mathrm{COX}$ reducing thromboxane $\mathrm{A} 2$ production and platelet aggregation. In high doses aspirin is a less effective analgesic agent than most NSAIDs. Hemodialysis is a useful treatment for aspirin elimination in case of overdose.

A 3 (D): The 6 independent predictors for major cardiac complications in Lee Revised Cardiac Risk Index (RCRI) for patients undergoing elective major non-cardiac surgery are:
i. High risk surgery
ii. History of ischemic heart disease
iii. History of congestive heart failure
iv. History of cerebrovascular disease
v. Preoperative insulin dependent diabetes mellitus

vi. Preoperative serum creatinine over 2.0 $\mathrm{mg} / \mathrm{dl}$

A 4 (B): Recommended PCI-to- any elective surgery interval is $>45$ days for bare metal stent and $>1$ year for drug eluting stent.

A 5 (D): Non-ST-segment elevation MI is confirmed by presence of elevated cardiac enzymes, in which the most specific one is troponin. The risk of reinfarction and cardiac death correlates with the magnitude of the rise in troponin.

A 6 (A): Thrombolytic therapy with tissue plasminogen activator (tPA), alteplase, reteplase, or tenecteplase should be initiated within 30-60 minutes of hospital arrival and within 12 hours of symptom onset. The most feared complication of thrombolytic therapy is intracranial hemorrhage. Contraindications to fibrinolytic therapy include hemorrhagic strokes within the previous year, known intracranial neoplasm, recent head trauma, active or recent internal bleeding (within 3 weeks), or suspected aortic dissection.

A 7 (A): The important prognostic determinants in patients with coronary artery disease are the anatomic extent of the atherosclerotic disease, the state of left ventricular function (ejection fraction), and the stability of the coronary plaque. Left main coronary artery disease is the most dangerous anatomic lesion 
and is associated with an unfavorable prognosis when managed with medical therapy alone. A stenosis of greater than $50 \%$ of the left main coronary artery is associated with an annual mortality rate of $15 \%$. The presence of vulnerable plaque (thin fibrous cap and large lipid core) predicts a greater risk of MI regardless of the degree of coronary artery stenosis.

A 8 (D): The present scenario supports diagnosis of ventricular septal rupture as a result of recent myocardial infarction (anterior). Septal rupture results in a left to right shunt with right ventricular volume overload increased pulmonary blood flow ad secondary left heart volume overload. A harsh systolic murmur is characteristic. Doppler echocardiography is diagnostic and excludes papillary muscle rupture which is a major differential diagnosis. The pulmonary artery catheter findings strongly suggest left to right shunt which are not explained by papillary muscle rupture. Medical management includes afterload reduction, diuretics and an intra-aortic balloon pump. Surgical repair is usually required.

A 9 (C): The simplest, most cost-effective method for detecting perioperative myocardial ischemia is standard ECG. Monitoring three leads (leads II, V4, and $V_{5}$, or else $V_{3}, V_{4}$, and $V_{5}$ ) may improve the ability to detect ischemia. The $\mathrm{V}_{5}$ lead (fifth intercostal space in the anterior axillary line) reflects myocardial ischemia in the portion of the left ventricle supplied by the left anterior descending coronary artery. Lead II is more likely to detect myocardial ischemia occurring in the distribution of the right coronary artery. Pulmonary artery catheter is a relatively insensitive method of monitoring for myocardial ischemia. If only small regions of left ventricular myocardium become ischemic, overall ventricular compliance and pulmonary artery occlusion pressure will remain unchanged.

A 10 (D): The transplanted heart has no sympathetic, parasympathetic, or sensory innervation, and the loss of vagal tone results in a higher-than-normal resting heart rate. The transplanted heart is unable to increase its heart rate immediately in response to hypovolemia or hypotension. It responds instead with an increase in stroke volume via the Frank-Starling mechanism. The heart rate increases in response to the effect of circulating catecholamines. Because $\alpha$ - and $\beta$ adrenergic receptors are intact on the transplanted heart 